\title{
Review of Structural Issues in the Design of a Box Wing Aircraft
}

\author{
Paul Jemitola and Paul Okonkwo*
}

\section{Air Force Institute of Technology, Nigerian Air Force, Nigeria}

Box wing aircraft weight issues, structural interactions as well as inter-wing joint designs differ remarkably from Conventional Cantilever Wing configuration and present design challenges which have been extensively investigated by several researchers. This study discusses the results of such investigations in order to highlight the prospects of the BoxWing aircraft as well as pending structural design challenges that require further examination.

\section{Introduction}

As part of the search for the next future airliner configuration, and to mitigate the negative impact of air travels on the environment, there have been renewed interests in the aircraft design of unconventional configurations such as the Blended Wing Body and Joined Wing aircraft concepts. The Joined/Box-Wing aircraft configuration attracted the attention of researchers due to its claimed merits of reduced structural weight and low induced drag [1]. Additionally, the potential of Joined/Box-Wing aircraft to improve fuel efficiency and reduce direct operating costs have been other reasons to investigate the configuration. Furthermore, the Joined/ Box-Wing aircraft configuration offers a lower risk than the Blended Wing Body concept because the departure from the conventional configuration is mainly with respect to the wing configuration. It is in view of these potentials that the National Aeronautics and Space Administration awarded a contract to Lockheed Martin to investigate the box wing aircraft configuration with the goal of reducing fuel burn by $40 \%$, nitrogen oxide emissions by $75 \%$ and noise by $42 \mathrm{~dB}$ [2].

Most of the literature on Joined/Box-Wing aircraft is credited to Wolkovitch [1] but prior to this time, work was carried out on similar concepts by Munk and Prandtl $[3,4]$. However, it was Wolkovitch [1] who published extensive literatures on the concept and is therefore quoted by most researchers of the Joined/Box-Wing aircraft configuration. Wolkovitch [1] views the Joined/Box-Wing aircraft configuration as a highly integrated concept that connects structural and aerodynamic properties in novel ways. This view is substantiated by Bernardini and Frediani [5] who also list the advantages of Joined/Box-Wing aircraft to include reduced structural weight, increased stiff ness, reduced wetted area, high trimmed maximum lift coefficient, low induced drag and reduced parasite drag. Furthermore, direct lift control, improved side-force control capability as well as good stability and control are other attributes of the concept.
As a consequence of the potentials of this configuration, a number of studies have been performed on the concept as a whole and on various aspects of the design.

Joined-Wing aircraft are known by different names such as Box wing, Bi-plane and Diamond wing aircraft. Though these configurations are often regarded as the same, this is not necessarily so. The essential differences in the various designs could be seen from the wing configuration and the principle of operation. As for the Joined and Box-Wing aircraft (see Figure 1 and Figure 2), the major difference in the two configurations is that while both wings produce equal amounts of lift [4] in a Box Wing aircraft, the fore wing produces about $80 \%$ [1] of the total lift in a classical JoinedWing aircraft. The focus of this paper is on the Joined-Wing aircraft that has tip ns connecting the tips of the fore and aft wings together and appropriately called a box wing aircraft.

\section{Weight Aspects}

Wolkovitch [1] states that Joined-Wing aircraft typically weighs $65 \%$ to $78 \%$ of the weight of aerodynamically equivalent conventional airplane. Bell [6] agrees by stating that the smaller wing span of a Joined-Wing aircraft's reduces the overall weight of the aircraft and minimises the bending moment on the wings. However, Wolkovitch [1] cautions that weight could only be saved if the geometric parameters of the Joined-Wing such as sweep, dihedral, taper ratio and joint location (as a fraction of span) are chosen properly. Furthermore, Wolkovitch [1] added that the relative weight advantage of the Joined-Wing aircraft compared to the conventional cantilever wing aircraft improves as wing sweep is reduced. Hajela's [7] study of the sensitivity of the structural weight of Joined-Wing aircraft to wing sweep and dihedral indicates that a wing sweep of about 17 degrees produces the lightest structure.

Weight is also reduced if the internal wing structure

*Corresponding author: Paul Okonkwo, Air Force Institute of Technology, Nigerian Air Force, Nigeria

Accepted: April 02, 2019

Published online: April 04, 2019

Citation: Jemitola P, Okonkwo P (2019) Review of Structural Issues in the Design of a Box Wing Aircraft. J Aerosp Eng Mech 3(1):161-166 


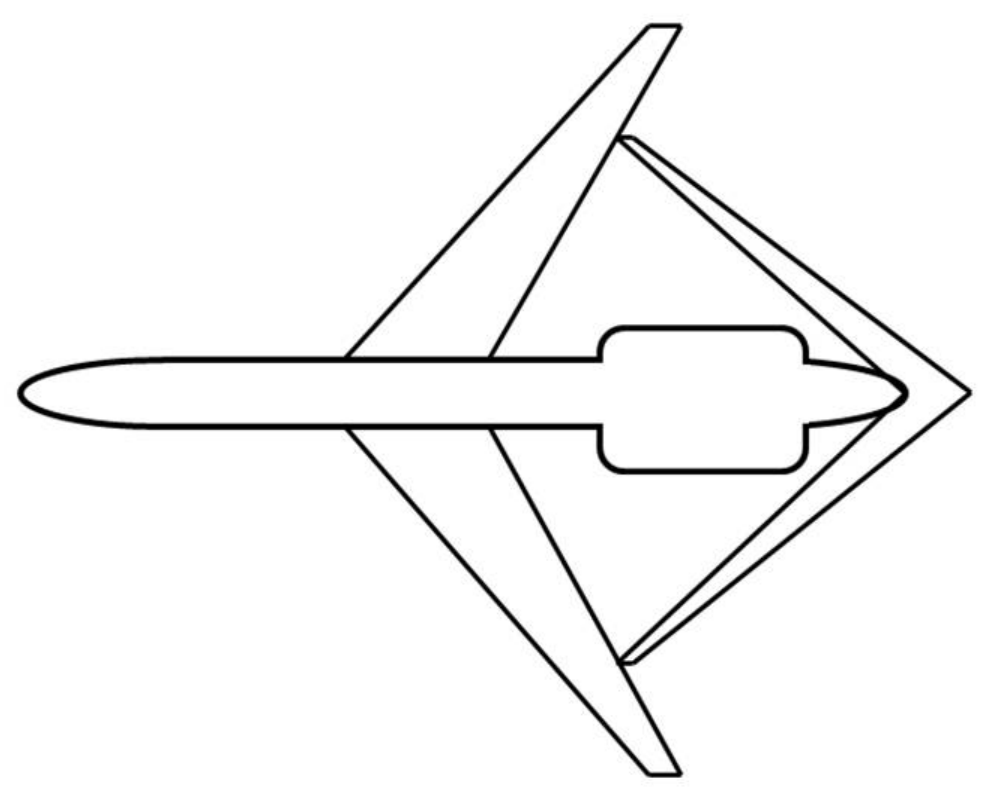

Figure 1: Joined-Wing Aircraft Sketch [13].

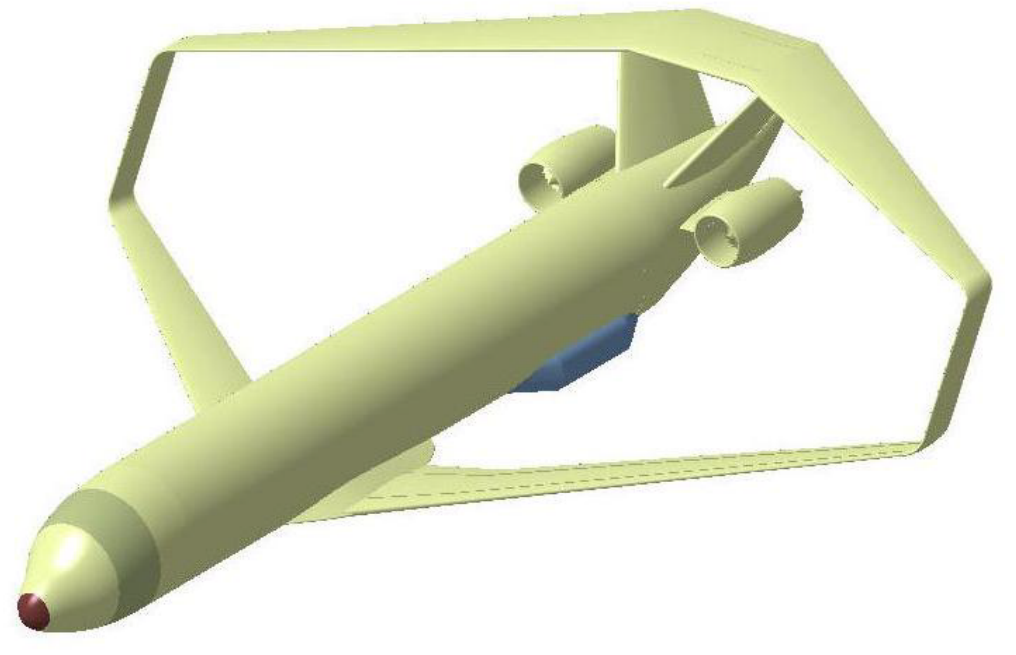

Figure 2: Box Wing Aircraft Sketch [13].

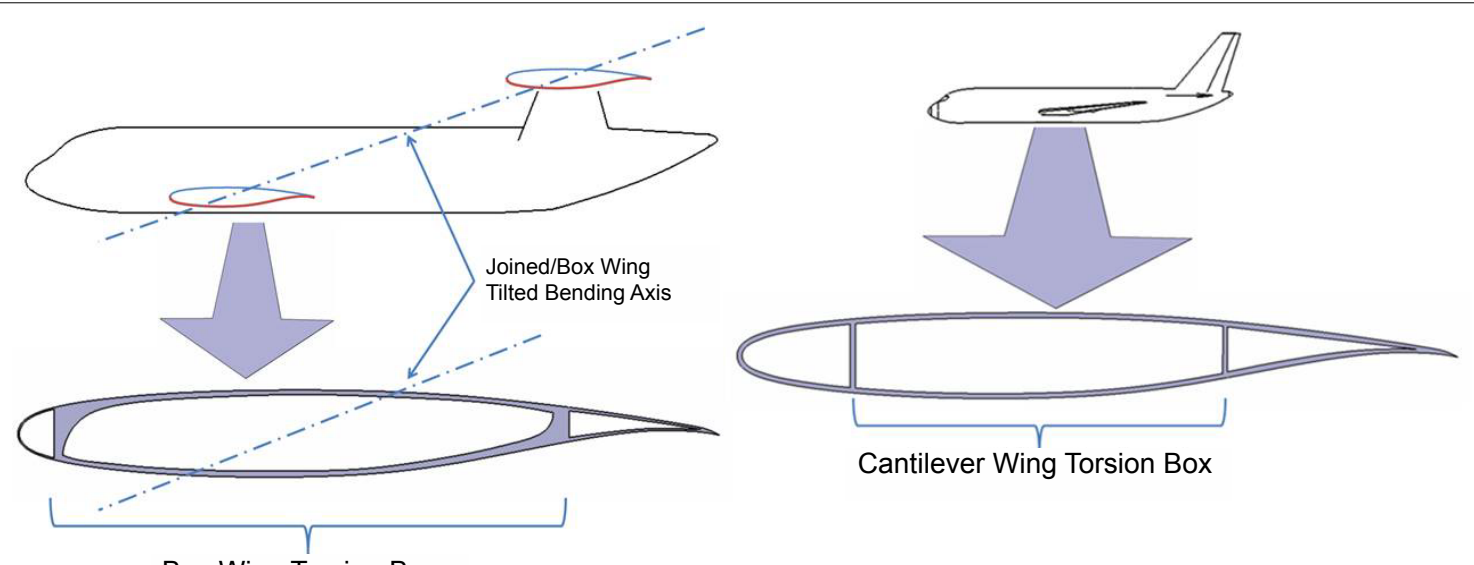

Box Wing Torsion Box

Figure 3: Wing Internal Structure [25]. 
of a Joined-Wing aircraft is optimized with the wing box occupying the section of the airfoil between $5 \%$ and $75 \%$ chord as sketched in Figure 3 [1]. Accordingly, Wolkovitch [1] recommends that the mean geometric chord of a Joined/BoxWing should be about $65 \%$ of the equivalent conventional cantilever wing aircraft. Wolkovitch [1] revealed that the inclined plane of a Joined-Wing aircraft will create a forward bending moment about the vertical axis. To counter this bending moment, Wolkovitch [1] states that the distribution of the structural elements should be as far away as possible from the inclined bending plane. This requires the upper leading edge and lower trailing edge of a Joined-Wing aircraft to contain the most structural material possible as highlighted in Figure 3. Blair and Canfield's [8] integrated design process for generating high fidelity analytical weight estimations for Joined-Wing concepts also showed that there are weight savings for this configuration compared to equivalent conventional airplane.

In a bid to reduce the structural weight of a Joined-Wing Aircraft, Lambert, Cooper and Nangia [9] developed the Buckling Alleviation Component. The method reduces the structural weight of the Joined-Wing Aircraft by finding an optimal balance between increasing the buckling load of the rear wing of the configuration and the resultant increase in the root bending moment of the front wing. Nevertheless, the Study finds that the tail height of a Joined-Wing Aircraft has the most significant effect on the structural weight of the configuration. Reduced tail height increases the amount of structural weight reduction obtained.

\section{Structural Aspects}

Wolkovitch [1] claims that the stiff ness of a Joined/ Box-Wing aircraft is high because the torsion of one wing is resisted by the flexure of the other. This enables a greater aileron effectiveness than is obtainable with conventional wings of similar weight. Bagwill and Selberg [10] concur by stressing the importance of having a wing gap to wing span ratio that is not greater than unity. This is necessary in order to keep the geometry as close to a reason-able truss-like structure as possible. Nevertheless, Patil [11] finds that stiff ness of a non-planar Joined-Wing aircraft is higher than the planar Joined-Wing aircraft.

Marisarla, et al. [12] undertook an investigation of the structural behaviour of a high altitude long endurance JoinedWing aircraft. By using loads obtained from computational fluid dynamics, non-linear and linear finite element analysis was performed. The study finds that the difference between values of maximum deflection derived from linear and nonlinear analysis was only about $2 \%$.

Jemitola, et al. [13] investigated the effect of tip $n$ inclination on the structural design of a Box-wing aircraft. Considering 5 tip $\mathrm{n}$ inclinations, the authors established that tip $\mathrm{n}$ inclination significantly affects the torsional force, dragwise shear force, and drag-wise bending moment distributions in the wings of a Box-wing aircraft. However, for out-of-plane bending moment and shear force distributions, only minor variations in wing structural design drivers as a function of tip $\mathrm{n}$ inclination were observed. Subsequently, Jemitola, et al. [13] recommend that utter and divergence analysis of the Box-wing configuration be performed for a more complete investigation of the effects of tip fin inclination on Box wing aircraft.

Wolkovitch [1] suggests that the fore and aft wing tips of a Joined-Wing aircraft should not overlap in plan view as this reduces aerodynamic efficiency. This view is supported by Bagwill and Selberg [10] as well as Frediani [14]. Wolkovitch [1] stressed that the fore wing of a Joined-Wing aircraft should be positioned forward of the fuselage and filleted appropriately.

Filleting is also recommended for the aft wing undersurface and vertical tail to minimize flow separation and interference drag [1]. As for wing twist, Wolkovitch [1] proposes wash-out for the fore wing and wash-in for the aft wing. He also states that it would be beneficial for the aft wing to incorporate less camber than the fore wing [1]. Bagwill and Selberg [10] views were corroborated by Wolkovitch [1] work on wash-out and wash-in for Joined-Wing aircraft.

Hajela and Chen [15] developed an approach for the optimal sizing of Cantilever and Joined-Wing structures by representing the built-up finite element model of the structures with an equivalent beam model. This approach enables the rapid estimation of the optimum structural weight of wing structures for a given geometry and a qualitative description of the material distribution. Sotoudeh and Hodges [16] introduced a new way of analyzing statically indeterminate structures as used on Joined-Wing aircraft. Sotoudeh and Hodges [16] formulation leads to the solution of a linear system of equations at each incremental loading step, thus avoiding the numerical difficulties associated with solving non-linear systems of equations. This includes finding suitable initial guesses needed for solving a Newton-Raphson equation of statically determinate and indeterminate structures.

Cesnik and Brown [17] assessed the prospect of using piezoelectric material technology to induce strain and produce wing-warping control on a Joined-Wing aircraft. This study was conducted based on a proposed framework which captures the non-linear defection behaviour of the wings, the effects of anisotropic piezoelectric composites embedded in the skin, and the unsteady subsonic aerodynamic forces acting on the wing. Cesnik and Brown [17] work showed the critical effect of the sudden loss of rear wing sti ness (or buckling) on vehicle integrity for Joined-Wing configuration.

Rasmussen, et al. [18] proposed a design methodology for Joined-Wing configurations that integrates a geometric model with other disciplinary models using the Adaptive Modelling Language. This creates a user interface that allows for the structural and aerodynamic analysis of the Joined-Wing configuration. Based on results from the design methodology, Rasmussen, et al. [18] conclude that non-linear structural analysis is important to accurately capture the large deformations that occur on a Joined-Wing configuration.

Muira, et al. [19] conducted a parametric weight evaluation of Joined-Wing aircraft by structural optimization. The study used a structural weight minimization capability for arbitrary wing configurations from the Programming 
Structural Synthesis System Software developed by the National Aeronautics and Space Administration. The purpose of the study was to present trends of the structural weight of Joined-Wing aircraft for conceptual/preliminary design of medium sized aircraft. Over 50 cases of structural weight minimizations were performed. The study showed that the structural weight of Joined-Wing aircraft depends strongly on wing geometry and structural arrangement. Furthermore, Muira, et al. [19] find that tip-joined Joined-Wing aircraft were approximately $36 \%$ lighter than an equivalent Conventional Cantilever wing aircraft.

Kalinowski [20] developed a code based on linear formulae that could be useful at the conceptual design stage to quickly optimize the structure of Box wing aircraft. The structural analysis was based on finite element beam models. The optimization performed was hybrid in nature using simple iteration and gradient descent methods. The Code highlighted several issues related to the interconnection of the lifting surfaces.

Gallman, et al. [21] note that buckling is a design issue for some Joined-Wing aircraft structures, especially 'Wolkovitch Joined-Wings', (Figure 1). Gallman, et al. [21] posit that Joined-Wing aircraft have structural efficiency but poor highlift capability. Additionally, Joined-Wing suffers a substantial penalty in maximum lift because of the attendant effect of having a short tail moment arm which subsequently required a large tail download required to trim in take-o configuration [21]. Consequently, Gallman, et al. [21] propose moving the centre of mass of the empty aircraft forward, thereby reducing the operational range of centre of mass. Gallman, et al. [21] also propose designing to a lower level of static stability as ways to reducing the take-off field length. Furthermore, redistributing the fuel and locating the engines on the wings could have a significant impact on take-off field length. The resulting effect on overall performance could reduce the direct operating cost of a Joined-Wing aircraft compared to the Conventional configuration. Therefore, Gallman, et al. [21] suggest that an extra fuel tank should be placed in the tail to trim the aircraft at the aft-most center of gravity.

\section{Inter-wing Joints}

One of the outcomes of Wolkovitch's [1] research is that locating the inter-wing joint at $70 \%$ of the fuselage length would give the lightest wing structure. Wolkovitch [1] also nds that tip-joined configuration is heavier than a conventional configuration of the equal span. Wolkovitch [1] added that reducing the span could decrease the structural weight of tip-joined configuration by $20 \%$ compared to an equivalent conventional Cantilever configuration. Wolkovitch [1] suggests that the structural weight could be minimised by decreasing the effective span/depth ratio, increasing the dihedral (positive and negative), lowering the sweep angles (positive and negative) and using a high taper ratios (fore and aft).

As for wing joint fixities (the attachment that connects the rear wing tips to the forward wing tips), Lin, et al. [22] examined 8 different joint fixity types using linear finite element and experimental method. The study concludes that the rigid wing joint has the best structural characteristics and hence is the most practicable for the classical Joined-Wing concepts shown in Figure 1 [22] However, Lin, et al. [22] advice that caution must be exercised when the results of their study are applied to other Joined/Box-Wing configurations and loading conditions.

Jemitola, et al. [23] performed a computational study to compare the stress distributions of 4 different wing/tip fin joint fixities using a finite element torsion box models of a Box-wing structure. The joint fixities refer to the type of attachment that connects the tip of the fore and aft wings to the end fin. Using loads from a vortex lattice tool, the analysis determined the best wing-joint fixity of a statically loaded idealized Box-wing configuration by comparing the stress distributions resulting from the different wing joints in addition to other essential aerodynamic requirements. Analysis of the wing joint fixities indicate that the rigid joint is the most suitable for a Joined-Wing aircraft.

Kimler and Canfield [18] studied the structural design of wing twist for pitch control of a Joined-Wing sensorcraft. The investigation involved adding a span-wise sliding joint into the wing structure of the vehicle's aft wing. The joint section where the forward and aft wings connect and form the outboard wing was also redesigned and analyzed to improve the load transmission between the wing spars. Using finite element methods, their results showed that the design of the inter-wing joint influenced the buckling resistance of the fore wing.

Weisshaar and Lee [24] explored the configuration changes of a Joined-Wing aircraft with respect to utter speed using Rayleigh-Ritz modelling, composite tailoring and linear optimization model. Varying the sweep angles from 30 to 45 degrees using parametric methods, Weisshaar and Lee [24] find that as the sweep angle rose for a fixed span size, the utter dynamic pressure also increases. However, as the joint location moved closer to the tip of the wing, the utter dynamic pressure decreases slightly.

Jemitola, et al. [25] developed a procedure for obtaining an empirical formula for estimating the masses of the fore and aft wings of a medium-range Box-wing aircraft. The procedure was based on Howe's [26] method for estimating the wing mass of Conventional Cantilever wing aircraft. Using a vortex lattice tool, finite element methods and regression analysis, modifications were performed on the coefficients in Howe's [27] method to enable its use on a medium range Box-wing aircraft. The results indicate that the fore and aftwings would use the same correction coefficient for their mass estimation. Furthermore, the study finds that the aftwing, due to its lower sweep angle, would be lighter than the fore-wing on a medium range Box-wing aircraft [25].

\section{Aero-elastic and Stability Considerations}

Kalinowski [28] undertook the aero-structural optimization of a Joined-Wing aircraft. Applying global search, modular algorithm based on automatic geometry generator, Finite Element Method and a Panel Method aerodynamic analysis solver, a $19 \%$ increase in total range was obtained from the 
baseline aircraft employed. Fazelzadeh, et al. [29] studied the 2D utter characteristics of a typical wing section of a Box Wing aircraft. Simulating the wing torsion and bending elasticity by torsional and longitudinal springs and applying the Theordorsen aerodynamic model, Fazelzadeh, et al. [29] observe that the tip fin stiff ness and angle have a significant effect on the aero-elastic utter boundary of a Box wing aircraft.

Perez and Andrew [30] developed a novel analytical model to assess the conditions required for the longitudinal stability of a Box wig aircraft. Taking into account the downwash from the fore and aft wings and considering the relative areas of each wing, stream-wise separations of the wing and the centre of gravity positions, the study finds that longitudinal stability is achieved by either reducing the area of the fore wing or by shifting the centre of gravity forward from the midpoints of the wings aerodynamic centre. Perez and Andrew [30] concludes that the unusual behaviour was caused by the asymmetric distribution of the down-wash upstream and downstream of the wing.

Dong Hwang-Lee [31] developed a methodology for aeroelastic tailoring and structural optimisation of the JoinedWing Configuration using the Purdue University Automated Structural Optimisation System. The study investigated the effect of aero-elastic constraints on the structural weight of isotropic and composite Joined-Wing. Additionally, the study analysed the aero-elastic features of a Joined-Wing Configuration using both the Rayleigh-Ritz Method and a Finite Element based aero-elastic stability and weight optimisation procedures. The study finds that increases in the fuselage pitching moment of inertia, increases the bodyfreedom utter speed. According to Dong Hwang Lee [31] there is a transition from body-freedom utter to cantilever utter when the pitching moment of inertial reaches a critical value.

\section{Conclusion}

The review of the structural aspects of the Joined/BoxWing aircraft highlights the unique features of this novel aircraft configuration as well as some challenges that require further studies. The study reviewed the weight characteristics, structural and inter-wing joint issues of a Joined/Box-Wing aircraft to ascerain the feasibility, advantages and disadvantages of the concept. The study nds that the JoinedWing aircraft typically weighs lower than an equivalent Conventional Cantilever Wing aircraft. Also, the study nds that optimal positioning of the inter-wing joints would lower the structural weight and improve the utter characteristics of the configuration. Furthermore, the study finds that rigid wing joint is the most appropriate joint fixity for classical Joined/Box-Wing aircraft.

\section{Acknowledgments}

The authors acknowledge the support of the Department of Aerospace Engineering, Cranfield University and Air Force Institute of Technology, Kaduna, Nigeria.

\section{References}

1. Wolkovitch J (1986) The joined-wing: An overview. Journal of Aircraft 23: 161-178.

2. https://www.flightglobal.com/news/articles/nasa-revealsthree-visions-for-a-future-airliner-351965/

3. Munk M (1923) The minimum induced drag of airfoils. Report 121. NACA.

4. Prandtl L (1924) Induced drag of Multiplanes. Technische Berichte 3: 309-315.

5. Bernardini G, Frediani A (1999) Aerodynamics for MDO of an innovative configuration. Applied Vehicle Technology Symposium on Aerodynamic Design and Optimization of Flight Vehicles in a Concurrent Multi-Disciplinary Environment. Research and Technology Organization, NATO.

6. Bell A, Fromm J, Lowery S, et al. (2008) Design and optimization of a joined-wing aircraft. University of Colorado, Boulder, Colorado, USA.

7. Hajela $P$ (1984) Weight Evaluation of the Joined-Wing Configuration. CR - 166592, NASA.

8. Blair M, Canfield R (2002) A joined-wing structural weight modelling study. 43rd AIAA/ASME/ASCE/AHS/ASC Structures, Structural Dynamics and Materials Conference, Colorado, USA.

9. Lambert LA, Cooper JE, Nangia RK (2016) Buckling alleviation for joined-wing aircraft. Journal of Aircraft 53: 811-821.

10. Bagwill T, Selberg B (1996) Aerodynamic investigation of joinedwing configurations for transport aircraft. AIAA 96-2373, 14th Appield Aerodynamics Conference, New Orleans, Louisiana.

11. Patil M (2003) Nonlinear aeroelastic analysis of joined-wing aircraft. AIAA-2003-1487, 44 ${ }^{\text {th }}$ AIAA/ASME/ASCE/AHS/ASC Structures, Structural Dynamics and Materials Conference, Norfolk, Virginia, USA.

12. Marisarla S, Narayanan V, Ghia U, et al. (2003) Prediction of structural behaviour of joined-wing configuration of high altitude long endurance aircraft, AIAA 2003-625, 41st Aerospace Sciences Meeting and Exhibit, Reno, Nevada, USA.

13. Jemitola PO, Montezino G, Fielding J, et al. (2012) Tip fin inclination effect on structural design of a box wing aircraft. Proceedings of the Institution of Mechanical Engineers, Part G: Journal of Aerospace Engineering.

14. Frediani A (2005) The prandtl wing. Lecture series on Innovative configurations and advanced concepts for future civil aircraft.

15. Hajela P, Chen J (1986) Optimum structural sizing of conventional cantilever and joined-wing configurations using equivalent bean models. AIAA-86-2653, AIAA/AHS/ASEE Aircraft Systems, Design and Technology Meeting, Dayton, Ohio, USA.

16. Sotoudeh Z, Hodges D (2011) Incremental method for structural analysis of joined-wing aircraft. Journal of Aircraft 48: 15881601.

17. Cesnik C, Brown E (2003) Active warping control of joined-wing airplane configuration. 44th AIAA/ASME/ASCE/AHS Structures, Structural Dynamics and Materials Conference, No 1715, Norfolk, Virginia.

18. Kimler FA, Canfield RA (2006) Structural design of wing twist for pitch control of joined-wing sensor aircraft. AIAA 2006-7134, 11th AIAA/ISSMO Multidisciplinary Analysis and Optimization Conference, Virginia, USA. 
19. Miura H, Shyu A, Wolkovitch V (1985) Parametric weight evaluation of joined-wings by structural optimization. AIAA/ ASME/ASCE/AHS 26th Structures, Structural Dynamics and Materials Conference, Florida.

20. Kalinowski MJ (2015) Structural optimization of box wing aircraft. Archive of Mechanical Engineering 62: 45-60.

21. Gallman J, Smith S, Kroo I (1993) Optimization of joined-wing aircraft. Journal of Aircraft 30: 897-905.

22. Lin H, Jhou J, Stearman R (1990) Influence of joint fixity on the structural static and dynamic response of a joined-wing aircraft. AIAA 90-0980, 31st AIAA/ASME/ASCE/AHS/ASC Structures Structural Dynamics and Materials Conference, Long Beach, California.

23. Jemitola PO, Fielding J, Stocking P (2012) Joint fixity effect on structural design of a box wing aircraft. The Aeronautical Journal 116: 363-372.

24. Weisshaar T, Lee D (2002) Aeroelastic tailoring of joined-wing configurations, AIAA 2002-1207, 43rd AIAA/ASME/ASCE/AHS/ ASC Structures, Structural Dynamics and Materials Conference, Denver, Colorado, USA.

25. Jemitola PO, Fielding J, Monterzino G (2012) Wing mass estimation algorithm for medium range box wing aircraft. Aeronautical Journal 117: 112-136.

26. Howe D (1996) The prediction of aircraft wing mass. Proc Instn Mech Engrs, Part G: Journal of Aerospace Engineering 210: 135145.

27. Howe D (2009) DAeT 9317. Aircraft Mass Prediction, Department of Aerospace Engineering, Cranfield University, Cranfield.

28. Kalinowski M (2017) Aero-structural optimisation of joined-wing aircraft. Transactions of the Institute of Aviation 249: 48-63.

29. Fazelzadeh, SA, Scholz, D, Mazidi A, et al. (2018) Flutter characteristics of typical wing sections of a box wing aircraft configuration. Advanced Aircraft Efficiency in a Global Air Transport System (AEGATS 2018), Toulouse France.

30. Andrews SA, Perez RE (2018) Analytic study of the conditions required for longitudinal stability of dual wing aircraft. Proceedings of the Institution of Mechanical Engineers, Part G: Journal of Aerospace Engineering 232: 213-246.

31. Lee DH (2002) Aeroelastic tailoring and structural optimization of joined-wing configurations. ProQuest Dissertations and Theses, 64: 3381. 